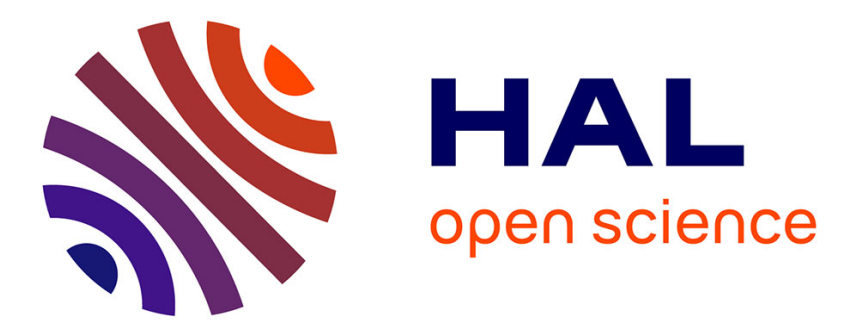

\title{
Problèmes de construction de type polynomial I - Caractérisations polynomiales des propriétés usuelles d'un plan
}

Frédéric Bertrand

\section{To cite this version:}

Frédéric Bertrand. Problèmes de construction de type polynomial I - Caractérisations polynomiales des propriétés usuelles d'un plan. 2008. hal-00277192v3

\section{HAL Id: hal-00277192 \\ https://hal.science/hal-00277192v3}

Preprint submitted on 12 May 2008

HAL is a multi-disciplinary open access archive for the deposit and dissemination of scientific research documents, whether they are published or not. The documents may come from teaching and research institutions in France or abroad, or from public or private research centers.
L'archive ouverte pluridisciplinaire HAL, est destinée au dépôt et à la diffusion de documents scientifiques de niveau recherche, publiés ou non, émanant des établissements d'enseignement et de recherche français ou étrangers, des laboratoires publics ou privés. 


\title{
Problèmes de construction de type polynomial I - Caractérisations polynomiales des propriétés usuelles d'un plan
}

\author{
FRÉDÉRIC BERTRAND* \\ Université Louis Pasteur \\ Institut de Recherche Mathématique Avancée ${ }^{\dagger}$
}

11 mai 2008

\begin{abstract}
Résumé
Un plan expérimental est solution d'un problème de construction de type polynomial si les coordonnées des points support du plan sont les solutions d'un système d'équations et d'inéquations polynomiales, système qui peut toujours être résolu à l'aide de la programmation semi-définie positive ou des bases de Gröbner. De nombreuses propriétés recherchées, comme l'optimalité alphabétique ou le blocage orthogonal, se formulent naturellement ainsi. Nous obtenons le même résultat pour la recherche de dispositifs $\mathcal{G}$-faiblement invariants, pour $\mathcal{G}$ un groupe de matrices compact quelconque. Pour citer cet article : F. Bertrand, C. R. Acad. Sci. Paris, Ser. I.
\end{abstract}

\begin{abstract}
Polynomial designs I - Polynomial characterizations of praised properties of a design

A design is said to be a polynomial design if the coordinates of the points of the design are the solutions of a system of polynomial equations or inequations; such a system can always be solved using semidefinite programming or Gröbner bases. Many praised properties of designs, such as alphabetic optimality and orthogonal blocking, can be easily stated in the framework of polynomial designs. The same holds true for $\mathcal{G}$-weakly invariant designs, $\mathcal{G}$ being any compact group of matrices. To cite this article: F. Bertrand, C. R. Acad. Sci. Paris, Ser. I.
\end{abstract}

\section{Abridged English version}

In this paper, we consider $\mathcal{G}$ a compact group of matrices and an experimental domain $\chi$ which is a compact subset, whose interior is not empty, of an Euclidean vector space of dimension $v$. We set, in section 3, the classical statistical

\footnotetext{
*Courriel : fbertran@math.u-strasbg.fr

$\dagger 7$ rue René Descartes, 67084 Strasbourg Cedex
} 
framework of full polynomial regression models of degree $d$ over the experimental domain $\chi$ as defined by equations (1) and (2). Then we recall the definitions of a design $\boldsymbol{\xi}$. As to the definitions of the $(\mathcal{G}, \mathcal{Q})$-equivariant property of a statistical model and the $\mathcal{G}$-weak invariance property we follow those of Gaffke and Heiligers, GH96.

We then deal with the set of invariant polynomials, denoted by $\mathbb{R}\left[x_{1}, \ldots, x_{v}\right]^{\mathcal{G}}$, for the natural action of a compact group $\mathcal{G}$ on the algebra of polynomials $\mathbb{R}\left[x_{1}, \ldots, x_{v}\right]$. It is a subalgebra of $\mathbb{R}\left[x_{1}, \ldots, x_{v}\right]$, see theorem 3.2 , which enables us to use computationnal commutative algebra, and especially Gröbner bases, [KR00] and [KR05, to assess whether a given polynomial is $\mathcal{G}$-invariant. Theorem 4.1 states that a design $\boldsymbol{\xi}$ is a $\mathcal{G}$-weakly invariant design if and only if the $E$-generating moment function, Ber08a], belongs to $\mathbb{R}\left[x_{1}, \ldots, x_{v}\right]^{\mathcal{G}}$, i.e. the coordinates of the points of the design are the solutions of a system of polynomial equations.

Searching the coordinates of the points of the design as the solution of a system of polynomial equations and inequations, is the general setting we define in 5.1 as the problem of finding polynomial designs. We show in the remaining of section 5 that using such a setting one can formulate many of the most wanted properties of a design. For instance one can search for the weak-invariance property, orthogonal blocking and alphabetic optimality, Kie74, the design belonging to an experimental domain $\chi$ of various shape, such as a ball, a cube or a simplex, either for finding new designs or for augmenting smartly existing ones. In section 6, we provide a methodology to derive polynomial designs or to prove that such designs do not exist using either Gröbner bases or semidefinite programming, Par03, and real algebraic geometry, Ste74. In order to reduce the amount of time required for computing the solutions of the system of polynomial equations and inequations, one can use designs built as the union of orbits of points by Coxeter groups, Kan01.

Additionnaly, the exact coordinates of the points of the design can be computed, which was done in Ber08b for many rotatable designs, BH57, in $\mathbb{R}^{3}$, allowing for the use of the tools provided by algebraic statistics, PRW00. Among these tools, one can find the way to gain full knowledge of the confoundings, to build lack of fit tests and to derive saturated models. Rotatable designs in $\mathbb{R}^{3}$ share praised properties both in response surface methodology, Puk93, and in threedimensional shape analysis, DMP05. Another interesting application of these setting and methodology that will be soon investigated by the author is to state results of non existence for some designs beginning with a conjecture of Hardin and Sloane, [HS96.

\section{Motivation}

Dans cette prépublication, nous nous intéressons à la construction de plans d'expériences satisfaisant à des propriétés fondamentales pour l'expérimentateur comme par exemple l'invariance faible, le blocage othogonal et l'optimalité alphabétique. Nous définissons pour cela un cadre particulier de construction que nous appelons problème de construction de type polynomial. Celui-ci est équivalent à la formulation obtenue par Stengle en 1974, Ste74, du théorème des zéros réels. Sa résolution peut alors être obtenue algorithmiquement en utilisant des outils récents d'algèbre computationnelle, [KR00] et [KR05], et de pro- 
grammation semi-définie positive, Par03. Lorsque la résolution algorithmique du problème est bien guidée, il est de surcroît possible d'obtenir les coordonnées exactes des points support du plan, permettant ainsi d'avoir recours aux outils de la statistique algébrique, [PRW00.

La réduction de la dimension d'un problème de constrution de type polynomial est une étape cruciale dans la résolution de celui-ci. Afin de proposer une stratégie permettant de réaliser celle-ci, nous nous appuyons sur des résultats qui ont été obtenus empiriquement par Hardin et Sloane, [HS96, dans le cas particulier de l'isovaraince. Pour un cardinal donné, parmi tous les plans sphériques isovariants certains forment des configurations régulières. Ce sont une union d'orbites d'un point par un sous-groupe d'un groupe de Coxeter, Kan01. Cette propriété ramène le problème de construction de dispositifs isovariants à celui du choix des points dont nous allions prendre l'orbite ainsi que du sous-groupe du groupe de réflexions à utiliser pour construire ces orbites. En utlisant cette approche, nous avons obtenu, dans [Ber08b], de nombreux exemples de résolution exacte de problèmes de construction de type polynomiale pour le cas particulier de l'isovariance et proposons de généraliser cette idée au cas d'un plus problème de construction de type polynomial quelconque.

\section{Notations, définitions et résultats préliminaires}

Nous nous intéressons à des modèles de régression linéaire soumis aux hypothèses statistiques usuelles. Nous considérons une variable explicative $\boldsymbol{x}$, dont les différentes valeurs possibles appartiennent à un domaine expérimental $\chi$, qui est une partie compacte d'un espace vectoriel de dimension finie $\mathbb{R}^{v}, v \in \mathbb{N}$ muni de sa structure euclidienne canonique, dont nous étudions l'influence sur une réponse $y$ à valeurs réelles. Soient $d$ un entier naturel et $A_{d}$ le sous-ensemble fini de $\mathbb{N}^{v}$ défini par $A_{d}=\left\{\boldsymbol{\alpha} \in \mathbb{N}^{v},|\boldsymbol{\alpha}| \leqslant d\right\}$, où $|\boldsymbol{\alpha}|$ désigne la somme des composantes du vecteur $\boldsymbol{\alpha}$. Les éléments de $A_{d}$ sont notés $\boldsymbol{\alpha}=\left(\alpha_{1}, \ldots, \alpha_{v}\right)$. Nous posons $k=\operatorname{Card}\left(A_{d}\right)=C_{d+v-1}^{v-1}$ et, pour $\boldsymbol{x} \in \mathbb{R}^{v}, \boldsymbol{f}(\boldsymbol{x})=\left(\boldsymbol{x}^{\boldsymbol{\alpha}}\right)_{\boldsymbol{\alpha} \in A_{d}}$ avec $\boldsymbol{x}^{\left(\alpha_{1}, \ldots, \alpha_{v}\right)}=x_{1}^{\alpha_{1}} \times \cdots \times x_{v}^{\alpha_{v}}$. Un modèle de régression multiple polynomiale complet de degré $d$, noté $A_{d}$, sur un domaine expérimental $\chi \in \mathbb{R}^{v}$ est alors défini par :

$$
y(\boldsymbol{x})=\boldsymbol{\theta}^{\prime} \boldsymbol{f}(\boldsymbol{x})=\sum_{\boldsymbol{\alpha}=\left(\alpha_{1}, \ldots, \alpha_{v}\right) \in A_{d}} \theta_{\boldsymbol{\alpha}} \prod_{i=1}^{v} x_{i}^{\alpha_{i}}, \quad \forall \boldsymbol{x}=\left(x_{1}, \ldots, x_{v}\right)^{\prime} \in \boldsymbol{\chi}
$$

où $\boldsymbol{\theta}^{\prime}=\left(\theta_{\boldsymbol{\alpha}}\right)_{\boldsymbol{\alpha} \in A_{d}} \in \mathbb{R}^{k}$, est un vecteur de paramètres inconnus. Il s'agit de la part déterministe d'un modèle stochastique défini de la manière suivante : l'observation des valeurs de la réponse $y$ aux points $\boldsymbol{x}_{\mathbf{1}}, \ldots, \boldsymbol{x}_{\boldsymbol{n}} \in \boldsymbol{\chi}$ est représentée par des variables aléatoires à valeurs réelles $Y_{1}, \ldots, Y_{n}$ telles que

$$
\begin{array}{rl}
i=1, \ldots, n & \mathbb{E}\left[Y_{i}\right]=y\left(\boldsymbol{x}_{\boldsymbol{i}}\right), \quad \mathbb{V} \operatorname{ar}\left[Y_{i}\right]=\sigma^{2}, \\
i, j=1, \ldots, n, i \neq j & \mathbb{C o v}\left[Y_{i}, Y_{j}\right]=0 .
\end{array}
$$

La variance constante $\left.\sigma^{2} \in\right] 0,+\infty[$ est inconnue et est de ce fait un paramètre additionnel du modèle.

Nous supposons que les valeurs pour lesquelles les observations sont effectuées 
sont connues exactement et contrôlées par l'expérimentateur : il s'agit du contexte usuel de la planification expérimentale.

Définition 3.1. Un plan approché $\boldsymbol{\xi}$ pour le modèle défini par les équations (1) et (2) est une mesure de probabilité de support fini sur $\boldsymbol{\chi}$, c'est-à-dire un couple $(\boldsymbol{X}, \boldsymbol{w}(\boldsymbol{X}))$ où $\boldsymbol{X}=\left\{\boldsymbol{x}_{\mathbf{1}}, \ldots, \boldsymbol{x}_{\boldsymbol{r}}\right\} \in \boldsymbol{\chi}$ est le support du plan et $\boldsymbol{w}(\boldsymbol{X})=$ $\left(w_{1}\left(\boldsymbol{x}_{\mathbf{1}}\right), \ldots, w_{r}\left(\boldsymbol{x}_{\boldsymbol{r}}\right)\right)$ sont les poids des points support $d u$ plan.

Nous commençons par quelques généralités sur les polynômes invariants par l'action d'un groupe de matrices. Nous renvoyons le lecteur à Hil82, Hum90. et Kan01 pour un exposé détaillé des propriétés des invariants polynomiaux dans le cas de l'action d'un groupe fini de matrices.

Proposition 3.1. Soit $\mathcal{G} \mathcal{L}_{v}(\mathbb{R})$ le groupe des matrices réelles inversibles d'ordre $v$. Le groupe $\mathcal{G} \mathcal{L}_{v}(\mathbb{R})$ opère sur l'agèbre de polynômes $\mathbb{R}\left[x_{1}, \ldots, x_{v}\right]$ de la manière suivante:

$$
\Theta_{\mathcal{G} \mathcal{L}_{v}(\mathbb{R})}:\left\{\begin{array}{ccc}
\mathcal{G} \mathcal{L}_{v}(\mathbb{R}) \times \mathbb{R}\left[x_{1}, \ldots, x_{v}\right] & \rightarrow & \mathbb{R}\left[x_{1}, \ldots, x_{v}\right] \\
(\boldsymbol{A}, P) & \mapsto & P(\boldsymbol{A x}),
\end{array}\right.
$$

ой $\boldsymbol{x}=\left(x_{1}, \ldots, x_{v}\right)^{\prime}$.

Proposition 3.2. Soit $\mathcal{G}$ un sous-groupe de $\mathcal{G} \mathcal{L}_{v}(\mathbb{R})$. Le groupe $\mathcal{G}$ opère sur l'agèbre de polynômes $\mathbb{R}\left[x_{1}, \ldots, x_{v}\right]$ de la manière suivante :

$$
\Theta_{\mathcal{G}}:\left\{\begin{array}{ccc}
\mathcal{G} \times \mathbb{R}\left[x_{1}, \ldots, x_{v}\right] & \rightarrow & \mathbb{R}\left[x_{1}, \ldots, x_{v}\right] \\
(\boldsymbol{A}, P) & \mapsto & P(\boldsymbol{A x})
\end{array}\right.
$$

où $\boldsymbol{x}=\left(x_{1}, \ldots, x_{v}\right)^{\prime}$.

Définition 3.1. Soit $\mathcal{G}$ un sous-groupe de $\mathcal{G} \mathcal{L}_{v}(\mathbb{R})$. Un polynôme $P$ de l'agèbre de polynômes $\mathbb{R}\left[x_{1}, \ldots, x_{v}\right]$ est $\mathcal{G}$-invariant si :

$$
\Theta_{\mathcal{G}}(g, P)=P, \forall g \in \mathcal{G} .
$$

Nous pouvons alors définir la $\mathbb{R}$-sous-algèbre de $\mathbb{R}\left[x_{1}, \ldots, x_{v}\right]$ constituée des polynômes qui sont invariants pour l'action de $\mathcal{G}$, un sous-groupe de $\mathcal{G} \mathcal{L}_{v}(\mathbb{R})$, obtenue par la restriction de l'action $\Theta_{\mathcal{G} \mathcal{L}_{v}(\mathbb{R})}$, introduite dans la définition 3.1 . à $\mathcal{G} \times \mathbb{R}\left[x_{1}, \ldots, x_{v}\right]$.

Définition 3.2. Soit $\mathcal{G}$ un sous-groupe de $\mathcal{G} \mathcal{L}_{v}(\mathbb{R})$. La $\mathbb{R}$-sous-algèbre de l'algèbre $\mathbb{R}\left[x_{1}, \ldots, x_{v}\right]$ constituée des polynômes $\mathcal{G}$-invariants, notée $\mathbb{R}\left[x_{1}, \ldots, x_{v}\right]^{\mathcal{G}}$, est :

$$
\mathbb{R}\left[x_{1}, \ldots, x_{v}\right]^{\mathcal{G}}=\left\{P \in \mathbb{R}\left[x_{1}, \ldots, x_{v}\right] \mid \Theta_{\mathcal{G}}(g, P)=P, \forall g \in \mathcal{G}\right\} .
$$

Remarque 3.1. Chaque élément de $\mathcal{G}$ est inversible ce qui implique que le degré total d'un polynôme est invariant pour l'action de $\mathcal{G}$. Nous en déduisons que la $\mathbb{R}$-sous-algèbre $\mathbb{R}\left[x_{1}, \ldots, x_{v}\right]^{\mathcal{G}}$ est une $\mathbb{R}$-sous-algèbre graduée de $\mathbb{R}\left[x_{1}, \ldots, x_{v}\right]$. De plus si un polynôme $P$ est homogène alors $\Theta_{\mathcal{G}}(g, P)$ est encore un polynôme homogène, pour tout élément $g$ de $\mathcal{G}$. Par conséquent nous pouvons restreindre l'action de $\mathcal{G}$ à tout espace vectoriel qui est somme d'espaces $\mathcal{H o m}_{i}(\mathbb{R})\left[x_{1}, \ldots, x_{v}\right]$, avec $i \in I \subset \mathbb{N}$ et $\mathcal{H o m}_{j}$ le sous espace vectoriel des polynômes homogènes de degré $j$ pour $j \in \mathbb{N}$. Par exemple nous pouvons restreindre l'action de $\mathcal{G}$ à $\mathbb{R}_{d}\left[x_{1}, \ldots, x_{v}\right]$ l'espace vectoriel des polynômes à $v$ indéterminées à coeffcients réels et de degré total inférieur ou égal à d. 
Nous introduisons maintenant les opérateurs de Reynolds pour l'action d'un groupe de matrices compact et avons pour cela besoin de la mesure de Haar. Ces opérateurs sont une généralisation de ceux introduits usuellemnt dans le cas de l'action d'un groupe de matrices fini, [Kan01].

Théorème 3.1. Soit $\mathcal{G}$ un groupe compact. Il existe une unique fonctionnelle linéaire $\mu$ définie sur $\mathcal{C}(\mathcal{G})$, le $\mathbb{C}$-espace vectoriel des fonctions continues $f$ : $\mathcal{G} \rightarrow \mathbb{C}$ continues, telle que $\mu$ est invariante à gauche et à droite pour l'action de $\mathcal{G}$ :

$$
\int_{\mathcal{G}} f(g x) \mu(\mathrm{d} x)=\int_{g \in \mathcal{G}} f(x g) \mu(\mathrm{d} x)=\int_{g \in \mathcal{G}} f(x) \mu(\mathrm{d} x), \quad \forall f \in \mathcal{C}(\mathcal{G})
$$

et

$$
\mu\left(1_{\mathcal{G}}\right)=1
$$

\section{Démonstration}

Nous renvoyons par exemple à [MT86] et à [Far06] ou à Wei65] pour un résultat plus général.

Définition 3.3. Soit $\mathcal{G} \subset \mathcal{G} \mathcal{L}_{v}(\mathbb{R})$ un groupe compact de matrices, l'opérateur de Reynolds associé à $\mathcal{G}$ est l'application $R_{\mathcal{G}}$ définie ainsi :

$$
\begin{aligned}
& R_{\mathcal{G}}: \mathbb{R}\left[x_{1}, \ldots, x_{v}\right] \quad \rightarrow \quad \mathbb{R}\left[x_{1}, \ldots, x_{v}\right] \\
& P(\boldsymbol{x}) \quad \mapsto \quad R_{\mathcal{G}}(P)=\int_{g \in \mathcal{G}} P(g \boldsymbol{x}) \mu(\mathrm{d} g),
\end{aligned}
$$

où $\mu$ est la mesure de Haar sur le groupe compact $\mathcal{G}$.

Remarque 3.1. Nous avons ici implicitement identifié fonctions polynomiales sur $\mathbb{R}^{v}$ et polynômes de $\mathbb{R}\left[x_{1}, \ldots, x_{v}\right]$. Ceci sera possible dans la suite dès que le domaine expériemental considéré sera par exemple d'intérieur non vide. Le symbole $\int_{g \in \mathcal{G}} P(g \boldsymbol{x}) \mu(\mathrm{d} g)$ de l'équation 10 est défini de la manière suivante. Le groupe $\mathcal{G}$ étant un groupe de matrice il préserve le degré total d'un polynôme. Nous restreignons alors l'action de $\mathcal{G}$ sur les fonctions polynomiales à celle sur les fonctions polynomiales homogènes de degré $d$ qui est sous-espace de dimension finie $\mathcal{G}$-stable. Considérons un polynôme $P=\sum_{\boldsymbol{\alpha} \in A} a_{\boldsymbol{\alpha}} \boldsymbol{x}^{\boldsymbol{\alpha}}$ homogène de degré $d$ et la fonction $\Omega_{P}$ définie par :

$$
\Omega_{P}:\left\{\begin{aligned}
\mathcal{G} \times \chi & \rightarrow \\
(g, \boldsymbol{x}) & \mapsto P(g(\boldsymbol{x}))=\sum_{\boldsymbol{\alpha}|| \boldsymbol{\alpha} \mid=d} f_{\boldsymbol{\alpha}}(g) \boldsymbol{x}^{\boldsymbol{\alpha}} .
\end{aligned}\right.
$$

La fonction $\Omega_{P}$ est continue sur $\mathcal{G} \times \chi$ pour la topologie usuelle puisque composée d'une fonction polynomiale homogène de degré $d$ et de la multiplication matricielle. Nous pouvons donc intégrer cette fonction par rapport à $g$ sur $\mathcal{G}$ en utilisant la mesure de Haar sur le groupe compact $\mathcal{G}$ introduite dans le théorème 3.1. Il suffit alors d'identifier la fonction polynomiale obtenue au polynôme de $\mathbb{R}\left[x_{1}, \ldots, x_{v}\right]$ correspondant.

\section{Proposition 3.3.}

Soit $R_{\mathcal{G}}$ l'opérateur de Reynolds associé au groupe compact de matrices $\mathcal{G}$.

-i- $R_{\mathcal{G}}$ est $\mathbb{R}$-linéaire. 


$$
\begin{aligned}
& \text {-ii- Si } P \in \mathbb{R}\left[x_{1}, \ldots, x_{v}\right]^{\mathcal{G}} \text { et si } Q \in \mathbb{R}\left[x_{1}, \ldots, x_{v}\right] \text {, alors } R_{\mathcal{G}}(P Q)=P R_{\mathcal{G}}(Q) \text {. } \\
& \text {-iii- L'image de } R_{\mathcal{G}} \text { est égale à } \mathbb{R}\left[x_{1}, \ldots, x_{v}\right]^{\mathcal{G}} \text {. } \\
& \text {-iv- L'opérateur de Reynolds est un projecteur }: R_{\mathcal{G}} \circ R_{\mathcal{G}}=R_{\mathcal{G}} \text {. }
\end{aligned}
$$

\section{Démonstration}

La première assertion est une conséquence de la linéarité de l'intégrale. La seconde assertion est une conséquence de la définition 3.1 de la $\mathcal{G}$-invariance. La troisième assertion et la quatrième assertion résultent de l'invariance de la mesure de Haar.

Nous indiquons maintenant un résultat concernant la structure de la $\mathbb{R}$-sousalgèbre $\mathbb{R}\left[x_{1}, \ldots, x_{v}\right]^{\mathcal{G}}$ de $\mathbb{R}\left[x_{1}, \ldots, x_{v}\right]$.

Théorème 3.2. Soit $\mathcal{G} \subset \mathcal{G} \mathcal{L}_{v}(\mathbb{R})$ un groupe de matrices compact. La $\mathbb{R}$-sousalgèbre $\mathbb{R}\left[x_{1}, \ldots, x_{v}\right]^{\mathcal{G}}$ de $\mathbb{R}\left[x_{1}, \ldots, x_{v}\right]$ est une $\mathbb{R}$-algèbre de type fini c'est-à-dire qu'il existe un nombre fini de polynômes homogènes $f_{1}, \ldots, f_{k}$ de $\mathbb{R}\left[x_{1}, \ldots, x_{v}\right]$, tous de degré $\geqslant 1$, tels que:

$$
\mathbb{R}\left[x_{1}, \ldots, x_{v}\right]^{\mathcal{G}}=\mathbb{R}\left[f_{1}, \ldots, f_{k}\right],
$$

où l'ensemble $\mathbb{R}\left[f_{1}, \ldots, f_{k}\right]$ est la sous-algèbre réelle de $\mathbb{R}\left[x_{1}, \ldots, x_{v}\right]$ engendrée par les poynômes $f_{1}, \ldots, f_{k}$.

\section{Démonstration}

Soit $I$ l'idéal de $\mathbb{R}\left[x_{1}, \ldots, x_{v}\right]$ engendré par tous les polynômes homogènes $P \in$ $\mathbb{R}\left[x_{1}, \ldots, x_{v}\right]^{\mathcal{G}}$ de degré total strictement positif. Soit $f_{1}, \ldots, f_{k}$ un système de générateurs homogènes de $I$. Nous remarquons que les $f_{1}, \ldots, f_{k}$ peuvent être choisis dans $\mathbb{R}\left[x_{1}, \ldots, x_{v}\right]^{\mathcal{G}}$. Supposons que l'ensemble $\mathbb{R}\left[x_{1}, \ldots, x_{v}\right]^{\mathcal{G}} \varsubsetneqq I$ et choisissons un polynôme $Q_{1} \in I \backslash \mathbb{R}\left[x_{1}, \ldots, x_{v}\right]^{\mathcal{G}}$ de degré minimal. Comme l'idéal $I$ est homogène, chacune des composantes homogènes de $Q_{1}$ est dans $I$. Par conséquent les composantes homogènes de $Q_{1}$ de degré strictementinférieur à celui de $Q_{1}$ appartiennent à $\mathbb{R}\left[x_{1}, \ldots, x_{v}\right]^{\mathcal{G}}$ par minimalité du degré de $Q_{1}$. Posons $Q$ la composante homogène de $Q_{1}$ de degré $Q_{1}$. Il existe des polynômes homogènes $h_{1}, \ldots, h_{k}$ de $\mathbb{R}\left[x_{1}, \ldots, x_{v}\right]$ tels que le polynôme $Q$ se décompose en $Q=\sum_{i=1}^{s} h_{i} f_{i}$ avec $\operatorname{deg} h_{i}>0$ et $\operatorname{deg} h_{i}+\operatorname{deg} f_{i}=\operatorname{deg} q$, voir par exemple [KR00. Nous appliquons alors l'opérateur de Reynolds à cette décomposition et nous déduisons de ses propriétés, voir la proposition 3.3 les égalités suivantes :

$$
\begin{aligned}
R_{\mathcal{G}}(Q) & =R_{\mathcal{G}}\left(\sum_{i=1}^{s} h_{i} f_{i}\right) \\
& =\sum_{i=1}^{s} R_{\mathcal{G}}\left(h_{i}\right) f_{i} \\
& =\sum_{i=1}^{s} h_{i} f_{i} \\
& =Q
\end{aligned}
$$

où nous avons, pour $i=1, \ldots, s$, les égalités $R_{\mathcal{G}}\left(h_{i}\right)=h_{i}$ puisque $\operatorname{deg} h_{i}<\operatorname{deg} Q$ et que $Q$ est un polynôme homogène de $I \backslash \mathbb{R}\left[x_{1}, \ldots, x_{v}\right]^{\mathcal{G}}$ de degré minimal. Appliquons maintenant le lemme 3.3 : le polynôme $Q=R_{\mathcal{G}}(Q)$ appartient à $\mathbb{R}\left[x_{1}, \ldots, x_{v}\right]^{\mathcal{G}}$ ce qui est en contradiction avec notre hypothèse. 
Remarque 3.2. Les générateurs $f_{1}, \ldots, f_{k}$ introduits dans le théorème 3.2 ne sont pas nécessairement algébriquement indépendants mêmes dans le cas d'un groupe fini de matrices. Le théorème de Shephard-Todd-Chevalley, voir par exemple [Hil82, Hum90] ou [Kan01, affirme, dans le cas d'un groupe fini $\mathcal{G}$, l'équivalence entre l'existence d'un isomorphisme de $\mathbb{R}$-algèbres entre $\mathbb{R}\left[x_{1}, \ldots, x_{v}\right]^{\mathcal{G}}$ et $\mathbb{R}\left[f_{1}, \ldots, f_{k}\right]$ et le fait que le groupe $\mathcal{G}$ est un groupe de pseudoréflexions. Nous serons donc dans cette situation dès que nous chercherons des invariances pour l'action d'un groupe de Coxeter sur $\mathbb{R}\left[x_{1}, \ldots, x_{v}\right]$.

Remarque 3.3. La détermination de tous les polynomes $\mathcal{G}$-invariants consiste donc à trouver les générateurs homogènes, $f_{1}, \ldots, f_{k}$ de $\mathbb{R}\left[x_{1}, \ldots, x_{v}\right]^{\mathcal{G}}$. Ceci peut être réalisé de manière algorithmique lorsque le groupe $\mathcal{G}$ est fini. Nous indiquons maintenant dans la proposition 3.4 l'une des manières d'y parvenir. Il existe des procédés plus raffinés, utilisant le théorème de Molien et la fonction génératrice de Hilbert en autres. Nous renvoyons par exemple à KR05 pour l'exposé de certains d'entre eux.

Proposition 3.4. Lorsque le groupe $\mathcal{G}$ est fini, l'algorithme suivant aboutit au calcul de polynômes homogènes $f_{1}, \ldots, f_{k}$ tels que:

$$
\mathbb{R}\left[x_{1}, \ldots, x_{v}\right]^{\mathcal{G}}=\mathbb{R}\left[f_{1}, \ldots, f_{k}\right] .
$$

- $i$ - Poser $\boldsymbol{L}=\emptyset$. Choisir un ordre $<$ sur les monômes de $\mathbb{R}\left[x_{1}, \ldots, x_{v}\right]$ compatible avec le degré total. Calculer le vecteur $\left(t_{1}, \ldots, t_{N}\right)$ formé des $N$ termes de degré plus petit que l'ordre du groupe $\mathcal{G},|\mathcal{G}|$, et ordonné de manière croissante pour l'ordre $<$.

-ii- Pour tout $l=1, \ldots, N$, calculer $R_{\mathcal{G}}\left(t_{l}\right)$. Utiliser le critère d'appartenance à une sous-algèbre, [KR00], pour décider si $R_{\mathcal{G}}\left(t_{l}\right) \in \mathbb{R}[P, P \in \boldsymbol{L}]$. Si cela n'est pas le cas ajouter $R_{\mathcal{G}}\left(t_{l}\right) \grave{a} \boldsymbol{L}$.

-iii- Renvoyer l'ensemble $\boldsymbol{L}$.

\section{Démonstration}

Nous renvoyons par exemple à [KR00] pour la preuve de cette proposition.

Remarque 3.4. Nous avons utilisé un ensemble de progammes Maple, le package Invar Kem93, pour obtenir la détermination complète de la structure des invariants polynomiaux pour l'action d'un groupe fini de matrices, c'est-à-dire aussi bien le calcul d'un système de générateurs homogènes de $\mathbb{R}$-sous-algèbre $\mathbb{R}\left[x_{1}, \ldots, x_{v}\right]^{\mathcal{G}}$, les invariants principaux, que celle d'un système de générateurs de l'idéal premier $I_{F}$ des relations, voir Kan01 pour des définitions de ces objets.

Corollaire 3.1. Soit $\mathcal{G}$ un groupe de matrices compact. Un polynôme $P$ est $\mathcal{G}$-invariant si et seulement si $P$ appartient à $\mathbb{R}\left[f_{1}, \ldots, f_{k}\right]$ avec $f_{i}, i=1, \ldots, k$, les polynômes homogènes de degré supérieur ou égal à 1 introduits dans le théorème 3.2.

Démonstration

Il s'agit d'une conséquence immédiate du théorème 3.2 . 
Remarque 3.5. La décision de l'appartenance d'un polynôme $P$ à la sousalgèbre $\mathbb{R}\left[f_{1}, \ldots, f_{k}\right]$ se traite de manière algorithmique à l'aide des bases de Gröbner, KR00 et KR05.

Nous concluons cette section par la caractérisation des polynômes invariants pour l'action du groupe orthogonal.

Proposition 3.5. Les polynômes de $\mathbb{R}\left[x_{1}, \ldots, x_{v}\right]$ invariants pour l'action $d u$ groupe orthogonal $\mathcal{O}_{v}$ sont les polynômes «radiaux»:

$$
\mathbb{R}\left[x_{1}, \ldots, x_{v}\right]^{\mathcal{O}_{v}}=\mathbb{R}\left[\sum_{i=1}^{v} x_{i}^{2}\right] .
$$

\section{Démonstration}

Nous renvoyons par exemple à [Ven01].

\section{Utilisation de la fonction $E$-génératrice des moments}

Nous avons introduit dans [Ber08a la fonction $E$-génératrice des moments d'un plan $\boldsymbol{\xi}$ lorsque le groupe $\mathcal{G}$ agit linéairement sur le domaine expérimental $\boldsymbol{\chi}$. Nous en avons déduit une caractérisation nécessaire et suffisante de l'invariance faible d'un plan par celle de l'invariance de la fonction $E$-génératrice des moments du plan lorsque le domaine expérimental $\chi$ est d'intérieur non vide. Ce résultat est toujours valide si les fonctions polynomiales appartenant au modèle forment un famille libre sur le domaine expérimental $\chi$ donc en particulier si la matrice des moments du plan n'est pas singulière.

Théorème 4.1. Considérons un domaine expérimental $\chi$ inclus dans $\mathbb{R}^{v}$ et d'intérieur non vide. Soit un modèle polynomial complet de degré $d(\mathcal{G}, \mathcal{Q})$-équivariant pour un groupe $\mathcal{G}$ qui agit linéairement sur $\chi$. Supposons que le groupe $\left\{U_{g}, g \in \mathcal{G}\right\}$, associé à l'action linéaire de $\mathcal{G}$ sur $\boldsymbol{\chi}$, est compact. Soit $E$ l'une des matrices symétriques réelles définies positives telle que le groupe $\left\{U_{g}, g \in \mathcal{G}\right\}$ est un sous-groupe de $\mathcal{O}_{v}(E)$, pour l'existence de $E$ voir [MT86]. Les assertions suivantes sont équivalentes :

-i- Le plan $\boldsymbol{\xi}$ est $\mathcal{G}$-faiblement invariant.

-ii- La fonction E-génératrice des moments, $\operatorname{MGF}_{E}^{A_{d}}(\boldsymbol{\xi})$, est $\mathcal{G}$-invariante.

-iii- La fonction E-génératrice des moments $\operatorname{MGF}_{E}^{A_{d}}(\boldsymbol{\xi})$ appartient à la sousalgèbre $\mathbb{R}\left[t_{1}, \ldots, t_{v}\right]^{\mathcal{G}}=\mathbb{R}\left[f_{1}, \ldots, f_{k}\right]$, où la $\mathbb{R}$-sous-algèbre $\mathbb{R}\left[t_{1}, \ldots, t_{v}\right]^{\mathcal{G}}$ de $\mathbb{R}\left[t_{1}, \ldots, t_{v}\right]$ a été introduite dans la définition 3.2 et les $f_{i}$, pour $i=$ $1, \ldots, k$, sont des polynômes homogènes tous de degré supérieur ou égal à 1 .

\section{Démonstration}

Un théorème de Ber08a] donne l'équivalence entre les deux premières assertions. La $E$-génératrice des moments, $\operatorname{MGF}_{E}^{A_{d}}(\boldsymbol{\xi})$, étant une fonction polynomiale sur $\mathbb{R}^{v}$ nous l'identifions avec la fonction polynomiale qui lui est canoniquement 
associée. En effet nous avons par définition de la fonction $E$-génératrice des moments :

$$
\operatorname{MGF}_{E}^{A_{d}}(\boldsymbol{\xi})(\boldsymbol{t})=\mathbb{E}_{\boldsymbol{\xi}}\left[\left(1+\langle\boldsymbol{t}, \boldsymbol{X}\rangle_{E}\right)^{2 d}\right], \forall \boldsymbol{t} \in \mathbb{R}^{v},
$$

où $\langle\boldsymbol{t}, \boldsymbol{x}\rangle_{E}=\boldsymbol{t}^{\prime} E \boldsymbol{x}$ pour tout $(\boldsymbol{t}, \boldsymbol{x})$ de $\mathbb{R}^{v} \times \mathbb{R}^{v}$. Nous appliquons alors le théorème 3.2 et le corollaire 3.1 ce qui nous permet de montrer que la deuxième et la troisième assertions sont équivalentes.

Nous souhaitons ramener la caractérisation polynomiale de l'invariance faible pour un sous-groupe compact de $\mathcal{G} \mathcal{L}_{v}(\mathbb{R})$ à celle de l'invariance d'une fonction génératrice des moments pour l'action d'un sous-groupe compact de $\mathcal{O}_{v}(\mathbb{R})$. Nous pourrons nous appuyer sur des résultats existants qui faciliteront grandement la détermination de la $\mathbb{R}$-sous-algèbre $\mathbb{R}\left[t_{1}, \ldots, t_{v}\right]^{\mathcal{G}}$.

Nous rappelons l'énoncé du théorème de [Ber08a] dont nous nous sommes servis pour réduire la contruction de plans expérimentaux $\mathcal{G}$-faiblement invariants pour l'action d'un groupe de matrices compact à celle de plans expérimentaux $\mathcal{K}$-faiblement invariants pour l'action d'un sous-groupe compact du groupe orthogonal.

Théorème 4.2. Soit un modèle polynomial complet de degré $d(\mathcal{G}, \mathcal{Q})$-équivariant pour un groupe $\mathcal{G}$ qui agit linéairement sur $\chi$. Supposons que le groupe $\left\{U_{g}, g \in \mathcal{G}\right\}$, associé à l'action linéaire de $\mathcal{G}$ sur $\boldsymbol{\chi}$, est compact. Soit A l'une des matrices inversibles telle que le groupe $\left\{U_{g}, g \in \mathcal{G}\right\}$ est conjugué à $\mathcal{K}$ un sous-groupe de $\mathcal{O}_{v}$, pour l'existence de $A$ voir [MT86]. La matrice $E=\left(A A^{\prime}\right)^{-1}$ est une matrice $E$ définie positive telle que $\mathcal{G}$ est un sous-groupe compact de $\mathcal{O}_{v}(E)$. Les assertions suivantes sont équivalentes :

-i- La fonction E-génératrice des moments, $\operatorname{MGF}_{E}^{A_{d}}(\boldsymbol{\xi})$, est $\mathcal{G}$-invariante.

-ii- La fonction génératrice des moments, $\mathrm{MGF}^{A_{d}}\left(\boldsymbol{\xi}^{A^{-1}}\right)$, est $\mathcal{K}$-invariante.

Nous en déduisons alors la caractérisation polynomiale de l'invariance faible énoncée dans le théorème 4.3 suivant.

Théorème 4.3. Considérons un domaine expérimental $\chi$ inclus dans $\mathbb{R}^{v}$ et d'intérieur non vide. Soit un modèle polynomial complet de degré $d(\mathcal{G}, \mathcal{Q})$-équivariant pour un groupe $\mathcal{G}$ qui agit linéairement sur $\chi$. Supposons que le groupe $\left\{U_{g}, g \in \mathcal{G}\right\}$, associé à l'action linéaire de $\mathcal{G}$ sur $\chi$, est compact. Soit A l'une des matrices inversibles telle que le groupe $\left\{U_{g}, g \in \mathcal{G}\right\}$ est conjugué à un sousgroupe de $\mathcal{O}_{v}$, pour l'existence de $A$ voir [MT86]. La matrice $E=\left(A A^{\prime}\right)^{-1}$ est une matrice $E$ définie positive telle que $\mathcal{G}$ est un sous-groupe compact de $\mathcal{O}_{v}(E)$. Les assertions suivantes sont équivalentes :

-i- Le plan $\boldsymbol{\xi}$ est $\mathcal{G}$-faiblement invariant.

-ii- La fonction génératrice des moments, $\mathrm{MGF}^{A_{d}}\left(\boldsymbol{\xi}^{A^{-1}}\right)$, est $\mathcal{K}$-invariante.

-iii- La fonction génératrice des moments, $\mathrm{MGF}^{A_{d}}\left(\boldsymbol{\xi}^{A^{-1}}\right)$ appartient à la sousalgèbre $\mathbb{R}\left[t_{1}, \ldots, t_{v}\right]^{\mathcal{K}}=\mathbb{R}\left[f_{1}, \ldots, f_{k}\right]$ de $\mathbb{R}\left[t_{1}, \ldots, t_{v}\right]$, où $\mathbb{R}\left[t_{1}, \ldots, t_{v}\right]^{\mathcal{K}}$ a été introduite dans la définition 3.2 et les $f_{i}$, pour $i=1, \ldots, k$, sont des polynômes homogènes tous de degré supérieur ou égal à 1 . 


\section{Démonstration}

Il s'agit d'une conséquence directe du théorème 4.1 et du théorème 4.2 .

Nous spécifions ces résultats pour un sous-groupe compact du groupe orthogonal $\mathcal{O}_{v}$.

Corollaire 4.1. Considérons un domaine expérimental $\chi$ inclus dans $\mathbb{R}^{v}$ et d'intérieur non vide. Soit un modèle polynomial complet de degré d $(\mathcal{G}, \mathcal{Q})$-équivariant pour un groupe $\mathcal{G}$ qui agit linéairement sur $\boldsymbol{\chi}$. Supposons que le groupe $\left\{U_{g}, g \in \mathcal{G}\right\}$, associé à l'action linéaire de $\mathcal{G}$ sur $\chi$, est un sous-groupe de $\mathcal{O}_{v}$. Les assertions suivantes sont équivalentes :

-i- Le plan $\boldsymbol{\xi}$ est $\mathcal{G}$-faiblement invariant.

-ii- La fonction E-génératrice des moments, $\mathrm{MGF}^{A_{d}}(\boldsymbol{\xi})$, est $\mathcal{G}$-invariante.

-iii- La fonction E-génératrice des moments $\operatorname{MGF}^{A_{d}}(\boldsymbol{\xi})$ appartient à la sousalgèbre $\mathbb{R}\left[t_{1}, \ldots, t_{v}\right]^{\mathcal{G}}=\mathbb{R}\left[f_{1}, \ldots, f_{k}\right]$, où la $\mathbb{R}$-sous-algèbre $\mathbb{R}\left[t_{1}, \ldots, t_{v}\right]^{\mathcal{G}}$ de $\mathbb{R}\left[t_{1}, \ldots, t_{v}\right]$ a été introduite dans la définition 3.2 et les $f_{i}$, pour $i=$ $1, \ldots, k$, sont des polynômes homogènes tous de degré supérieur ou égal à 1.

\section{Démonstration}

Puisque le groupe $\mathcal{G}$ est un sous-groupe compact de $\mathcal{O}_{v}$ nous pouvons appliquer le théorème 4.3 en choisissant $A=I_{v}$.

Remarque 4.1. Il est fréquent de chercher des dispositifs qui présentent la propriété de $\mathcal{G}_{s p}$-invariance faible. Cette invariance par rapport au groupe hyperoctaédral, qui est fini donc compact pour la topologie usuelle sur $\mathcal{M}_{n}(\mathbb{R})$, est également qualifiée de $\mathcal{B}_{m}$-invariance faible. Le théorème 4.1 montre donc l'équivalence entre la $\mathcal{B}_{m}$-invariance faible du plan et la $\mathcal{B}_{m}$-invariance de la fonction génératrice des moments.

Corollaire 4.2. Considérons un domaine expérimental $\chi$ inclus dans $\mathbb{R}^{v}$ et d'intérieur non vide. Soit un modèle polynomial complet de degré d $(\mathcal{G}, \mathcal{Q})$-équivariant pour un groupe $\mathcal{G}$ qui agit linéairement sur $\chi$. Supposons que le groupe $\left\{U_{g}, g \in \mathcal{G}\right\}$, associé à l'action linéaire de $\mathcal{G}$ sur $\boldsymbol{\chi}$, est un groupe compact maximal de $\mathrm{GL}_{n}(\mathbb{R})$. Soit $A$ l'une des matrices inversibles telle que le groupe $\left\{U_{g}, g \in \mathcal{G}\right\}$ est conjugué à un sous-groupe de $\mathcal{O}_{v}$, pour l'existence de $A$ voir [MT86]. La matrice $E=\left(A A^{\prime}\right)^{-1}$ est une matrice $E$ définie positive telle que $\mathcal{G}$ est un sous-groupe compact de $\mathcal{O}_{v}(E)$. Les assertions suivantes sont équivalentes :

- $i$ - Le plan $\boldsymbol{\xi}$ est $\mathcal{G}$-faiblement invariant.

-ii- La fonction génératrice des moments, $\operatorname{MGF}^{A_{d}}\left(\boldsymbol{\xi}^{A^{-1}}\right)$, est $\mathcal{O}_{v}$-invariante.

-iii- La fonction génératrice des moments, $\mathrm{MGF}^{A_{d}}\left(\boldsymbol{\xi}^{A^{-1}}\right)$ appartient à la sousalgèbre $\mathbb{R}\left[\sum_{i=1}^{v} x_{i}^{2}\right]$ des polynômes radiaux.

\section{Démonstration}

Si le groupe $\mathcal{G}$ est un sous-groupe compact maximal de $\mathcal{G} \mathcal{L}_{v} \mathbb{R}$ il est conjugué à $\mathcal{O}_{v}$ tout entier car $\mathcal{O}_{v}$ est un sous-groupe compact maximal de $\mathcal{G} \mathcal{L}_{v} \mathbb{R}$. Nous appliquons alors le théorème 4.3 puis la proposition 3.5 ce qui permet d'aboutir 
au résultat recherché.

Nous spécifions ces résultats dans le cas de l'isovariance :

Corollaire 4.3. Considérons un domaine expérimental $\chi$ inclus dans $\mathbb{R}^{v}$ et d'intérieur non vide. Considérons un domaine expérimental $\boldsymbol{\chi}$ inclus dans $\mathbb{R}^{v}$, un modèle polynomial de degré $d\left(\mathcal{O}_{\text {orth }}, \mathcal{Q}_{\text {orth }}\right)$-équivariant et un plan expérimental $\boldsymbol{\xi}$ dont le support est inclus dans $\boldsymbol{\chi}$.

- $i$ - Le plan $\boldsymbol{\xi}$ est isovariant.

-ii- La fonction génératrice des moments, $\mathrm{MGF}^{A_{d}}(\boldsymbol{\xi})$, est $\mathcal{O}_{v}$-invariante.

-iii- La fonction génératrice des moments, $\mathrm{MGF}^{A_{d}}(\boldsymbol{\xi})$ appartient à la sousalgèbre $\mathbb{R}\left[\sum_{i=1}^{v} x_{i}^{2}\right]$ des polynômes radiaux.

\section{Démonstration}

Nous déduisons de la proposition 3.5 l'égalité :

$$
\mathbb{R}\left[x_{1}, \ldots, x_{v}\right]^{\mathcal{O}_{v}}=\mathbb{R}\left[\sum_{i=1}^{v} x_{i}^{2}\right] .
$$

Une application du théorème 4.3 permet alors de conclure.

Nous pouvons utiliser le corollaire 4.4 suivant pour identifier directement des dispositifs isovariants.

Corollaire 4.4. Considérons un domaine expérimental $\chi$ inclus dans $\mathbb{R}^{v}$ et d'intérieur non vide, un modèle polynomial de degré $d(\mathcal{G}, \mathcal{Q})$-équivariant pour $\mathcal{G}$ un sous-groupe du groupe orthogonal $\mathcal{O}_{v}$ et un plan expérimental $\boldsymbol{\xi}$ dont le support est inclus dans $\chi$. Soit $\mathcal{D}=\left(d_{1}, \ldots, d_{t}\right)$ la suite finie des degrés des invariants principaux du groupe $\mathcal{G}$ inférieurs ou égaux à $2 d$. Si $\mathcal{D}$ est réduite à $\left(d_{1}=2\right)$ alors le plan est isovariant.

\section{Démonstration}

Une application du corollaire 4.1 indique que la fonction génératrice des moments $\operatorname{MGF}^{A_{d}}(\boldsymbol{\xi})$ appartient à $\mathbb{R}\left[x_{1}, \ldots, x_{v}\right]^{\mathcal{G}}$. Or cette $\mathbb{R}$-sous-algèbre d'invariants est égale à $\mathbb{R}\left[f_{1}, \ldots, f_{k}\right]$ par application du théorème 3.2 . Nous pouvons supposer que les $f_{i}$, pour $i=1, \ldots, k$, sont ordonnés par degré total croissant. L'hypothèse du corollaire implique alors que le seul polynôme de degré supérieur ou égal à 1 et inférieur ou égal à $2 d$ est un polynôme en $f_{1}$ avec $f_{1}$ de degré 2. Puis le polynôme $\sum_{i=1}^{v} x_{i}^{2}$ est un polynôme $\mathcal{G}$-invariant de degré 2 puisqu'il est $\mathcal{O}_{v}$-invariant par la proposition 3.5. Ainsi la fonction génératrice des moments $\mathrm{MGF}^{A_{d}}(\boldsymbol{\xi})$ est un polynôme en $\sum_{i=1}^{v} x_{i}^{2}$ et appartient alors en fait à $\mathbb{R}\left[x_{1}, \ldots, x_{v}\right]^{\mathcal{O}_{v}}$. Une application du corollaire 4.3 permet alors de conclure.

Ainsi l'utilisation de la fonction génératrice des moments nous permet de nous ramener, dans le cas de l'étude d'un plan appartenant à domaine expérimental d'intérieur non vide ou pour lequel les fonctions du modèle forment une famille libre, à des caractérisations d'invariance polynomiales pour un groupe compact $\mathcal{G}$ ou pour un sous-groupe de $\mathcal{O}_{v}$ auquel il est conjugué. 


\section{Problèmes de construction de type polynomial}

Nous commençons par définir un problème de construction de type polynomial d'un plan expérimental.

Définition 5.1. Un problème de construction de type polynomial d'un plan expérimental $\boldsymbol{\xi}$ de support $\boldsymbol{X}$, un sous-ensemble de $\mathbb{R}^{v}$ de cardinal $r$, est défini de la manière suivante :

- $i$ - Une liste $\mathcal{O} b j$ d'objectifs à remplir. Ces objectifs peuvent être de trois types et de priorité variable :

(i) Appartenance à un domaine expérimental $\chi$ inclus dans $\mathbb{R}^{v}$ fixé à l'avance caractérisé par les inéquations polynomiales

$$
\left\{\begin{aligned}
g_{1}(\boldsymbol{x}) & \leqslant 0 \\
& \vdots \\
g_{u}(\boldsymbol{x}) & \leqslant 0,
\end{aligned}\right.
$$

c'est-à-dire $\chi=\left\{\boldsymbol{x} \in \mathbb{R}^{v} \mid g_{i}(\boldsymbol{x}) \leqslant 0,1 \leqslant i \leqslant u\right\}$.

(ii) Les points support du plan $\boldsymbol{\xi}$ sont les solutions d'un système d'équations polynomiales $f_{1}(\boldsymbol{x})=\ldots=f_{s}(\boldsymbol{x})=0$ associé à l'idéal $I=$ $\left\langle f_{1}, \ldots, f_{s}\right\rangle$ de $\mathbb{R}\left[x_{1}, \ldots, x_{v}\right]$, c'est-à-dire que les points support $d u$ plan $\boldsymbol{\xi}$ appartiennent à une variété algébrique $\mathbf{V}(I)$ réelle fixée.

(iii) Minimisation de critères polynomiaux $h_{1}, \ldots, h_{t}$ en les coordonnées des points support du plan $\boldsymbol{\xi}$.

-ii- Un ensemble de $r_{0}$ points supports du plan $\boldsymbol{X}_{\mathbf{0}}$, fixés par l'expérimentateur et dont les coordonnées sont connues de manière exacte, qui doivent nécessairement appartenir au support $\boldsymbol{X}$ du plan $\boldsymbol{\xi}$.

-iii- Un nombre, égal à $r-r_{0}$, de points dont nous souhaitons déterminer les coordonnées de manière exacte de telle sorte que le plan expérimental remplisse les objectifs $\mathcal{O} b j$.

Nous utiliserons principalement des techniques liées aux bases de Gröbner, KR00 et KR05, ou à la programmation semi-définie positive, Par03, pour résoudre ces problèmes qui sont étroitement liés au théorème des zéros réel, Stu02. Par résolution nous entendons soit l'obtention des coordonnées exactes des points support du plan soit la preuve qu'il n'existe pas de dispositif satisfaisant aux objectifs spécifiés.

Définition 5.2. Un critère d'optimalité est dit polynomial si l'une de ces puissances est un polynôme en les coordonnées des points du plan.

Proposition 5.1. Les critères $\phi_{p}$, pour $p$ entier positif, Kier74, donc le critère de D-optimalité, et le critère de I-optimalité, [GH96], sont des critères d'optimalité polynomiaux.

\section{Démonstration}

Il s'agit d'une conséquence immédiate de la définition. 


\section{Remarque 5.1.}

Le premier objectif, l'appartenance des points support du plan à un domaine expérimental $\chi$ inclus dans $\mathbb{R}^{v}$ fixé à l'avance caractérisé par les inéquations polynomiales $g_{1}(\boldsymbol{x}) \leqslant 0, \ldots, g_{u}(\boldsymbol{x}) \leqslant 0$, couvre les cas suivants :

-i- Une boule centrée ou non à l'origine de $\mathbb{R}^{v}$ muni du produit scalaire canonique si les coordonnées de chacun des points support du plan sont solution de $\sum_{i=1}^{v} x_{i}^{2}-r \leqslant 0$ avec $r \geqslant 0$.

-ii- Un ellipsoïde centré ou non à l'origine de $\mathbb{R}^{v}$ muni du produit scalaire canonique si les coordonnées de chacun des points support du plan sont solution de $\sum_{i=1}^{v} a_{i}^{2} x_{i}^{2}-r \leqslant 0$, avec $a_{i}>0$, pour $i=1, \ldots, v$ et $r \geqslant 0$.

-iii- Plus généralement une boule centrée ou non à l'origine de $\mathbb{R}^{v}$ muni d'un produit scalaire quelconque $\langle\cdot, \cdot\rangle_{E}$ associé à la forme quadratique $q_{E}$ si les coordonnées de chacun des points support du plan sont solution de $q_{E}\left(x_{1}, \ldots, x_{v}\right) \leqslant r$ avec $r \geqslant 0$.

-iv- Un simplexe centré ou non à l'origine de $\mathbb{R}^{v}$ puisqu'il est décrit comme l'intersection de demi-espaces affines. Les coordonnées de chacun des points support du plan devant alors être solution du système d'inéquations affines associé.

-v- Un cube centré ou non à l'origine de $\mathbb{R}^{v}$ comme l'intersection de demiespaces affines. Les coordonnées de chacun des points support du plan devant alors être solution de du système d'inéquations affines associé.

Nous retrouvons dans cette énumération les formes de domaines expérimentaux les plus couramment utilisées en planification expérimentale.

\section{Remarque 5.2.}

Le second objectif, les points support du plan $\boldsymbol{\xi}$ sont les solutions d'un système d'équations polynomiales $f_{1}(\boldsymbol{x})=\ldots=f_{s}(\boldsymbol{x})=0$ associé à l'idéal $I=\left\langle f_{1}, \ldots, f_{s}\right\rangle$ de $\mathbb{R}\left[x_{1}, \ldots, x_{v}\right]$, c'est-à-dire que les points support du plan $\boldsymbol{\xi}$ appartiennent à une variété algébrique $\mathbf{V}(I)$ réelle fixée, permet de considérer les domaines expérimentaux suivants :

-i- Une sphère centrée ou non à l'origine de $\mathbb{R}^{v}$ muni du produit scalaire canonique pour $\sum_{i=1}^{v}\left(x_{i}-c_{i}\right)^{2}-r=0$ avec $c_{i}>0$, pour $i=1, \ldots, v$ et $r \geqslant 0$.

-ii- La frontière d'un ellipsoïde centré ou non à l'origine de $\mathbb{R}^{v}$ muni du produit scalaire canonique pour $\sum_{i=1}^{v} a_{i}^{2}\left(x_{i}-c_{i}\right)^{2}-r=0$, avec $a_{i}>0$, pour $i=1, \ldots, v, c_{i} \in \mathbb{R}$, pour $i=1, \ldots, v$, et $r \geqslant 0$.

-iii- Plus généralement une sphère centrée ou non à l'origine de $\mathbb{R}^{v}$ muni d'un produit scalaire quelconque $\langle\cdot, \cdot\rangle_{E}$ associé à la forme quadratique $q_{E}$ pour $q_{E}\left(x_{1}, \ldots, x_{v}\right) \leqslant r$ avec $r \geqslant 0$.

Nous retrouvons ici le cas de la sphère et d'autres domaines expérimentaux d'intérieur vide.

Remarques 5.1.

Le problème de construction d'un plan expérimental appartenant à un domaine expérimental $\chi$, inclus dans $\mathbb{R}^{v}$ fixé à l'avance caractérisé par les inéquations polynomiales $g_{1}(\boldsymbol{x}) \leqslant 0, \ldots, g_{u}(\boldsymbol{x}) \leqslant 0$, est un problème de construction de type polynomial, tel que nous l'avons énoncé dans la définition 5.1, dans les cas particuliers suivants que nous développerons dans le paragraphe indiqué : 
-i- Construction de plans faiblement invariants pour l'action d'un groupe de matrices compact, $c f .5 .1$.

-ii- Construction de plans optimaux pour un critère polynomial, $c f .5 .2$

-iii- Construction de plans partiellement invariants pour l'action d'un groupe de matrices compact et optimaux pour un critère polynomial, $c f .5 .3$.

-iv- Construction de plans bloqués orthogonalement, $c f$. 5.4 .

-v- Augmentation de plans invariants pour l'action d'un groupe de matrices compact, $c f .5 .1$.

-vi- Augmentation de plans optimaux pour un critère polynomial, $c f .5 .2$

-vii- Augmentation de plans partiellement invariants pour l'action d'un groupe de matrices compact et optimaux pour un critère polynomial, $c f .5 .3$.

-viii- Augmentation de plans bloqués orthogonalement, $c f$. 5.4 .

Remarques 5.2. Par rapport au contexte usuel de la recherche de plans expérimentaux optimaux nous nous sommes imposés la condition supplémentaire de la détermination exacte des coordonnées des points support du plan. Ainsi les algorithmes numériques couramment utilisés par les planificateurs ne peuvent s'appliquer dans notre contexte. Il s'agit par exemple de l'algorithme de construction de plans expérimentaux $D$-optimaux de Federov-Wynn [NM92, du recuitsimulé [CMMR87 ou de l'algorithme du simplexe GW00. Nous renvoyons à [BOB07] pour un exemple d'application, réalisé par l'auteur, de ces algorithmes à des données réelles. Il en va de même en ce qui concerne la construction de plans isovariants par des méthodes numériques comme celles proposées par Hardin et Sloane dans leur logiciel Gosset, HS91. Toutefois nous verrons dans la prépublication [Ber08b] comment il nous est possible d'utiliser leurs résultats numériques pour guider, en la simplifiant, notre méthodologie de construction exacte.

\subsection{Construction ou augmentation faiblement-invariante d'un plan expérimental}

Les résultats que nous avons obtenus dans la section 3 permettent de montrer que la construction ou l'augmentation d'un plan expérimental est un problème de construction de type polynomial, comme introduit dans la définition 5.1. Nous renvoyons à la prépubication Ber08b pour plusieurs applications concrètes.

Théorème 5.1. Considérons un domaine expérimental $\chi$ inclus dans $\mathbb{R}^{v}$, caractérisé par les inéquations polynomiales $g_{1}(\boldsymbol{x}) \leqslant 0, \ldots, g_{u}(\boldsymbol{x}) \leqslant 0$ et d'intérieur non vide. Soit un modèle polynomial complet de degré $d(\mathcal{G}, \mathcal{Q})$-équivariant pour un groupe $\mathcal{G}$ qui agit linéairement sur $\boldsymbol{\chi}$. Supposons que le groupe $\left\{U_{g}, g \in \mathcal{G}\right\}$, associé à l'action linéaire de $\mathcal{G}$ sur $\boldsymbol{\chi}$, est compact. Le problème de la construction, si $\boldsymbol{X}_{0}=\emptyset$, ou de l'augmentation, si $\boldsymbol{X}_{0} \neq \emptyset$, d'un plan expérimental $\boldsymbol{\xi}$ est équivalent à un problème de construction de type polynomial.

\section{Démonstration}

Nous appliquons le théorème 4.1 dont les conditions d'utilisation sont remplies. Soit $E$ l'une des matrices symétriques réelles définies positives telle que le groupe $\left\{U_{g}, g \in \mathcal{G}\right\}$ est un sous-groupe de $\mathcal{O}_{v}(E)$, pour l'existence de $E$ voir [MT86]. Les assertions suivantes sont donc équivalentes : 
-i- Le plan $\boldsymbol{\xi}$ est $\mathcal{G}$-faiblement invariant.

-ii- La fonction $E$-génératrice des moments, $\operatorname{MGF}_{E}^{A_{d}}(\boldsymbol{\xi})$, est $\mathcal{G}$-invariante.

-iii- La fonction $E$-génératrice des moments $\operatorname{MGF}_{E}^{A_{d}}(\boldsymbol{\xi})$ appartient à l'algèbre $\mathbb{R}\left[t_{1}, \ldots, t_{v}\right]^{\mathcal{G}}=\mathbb{R}\left[f_{1}, \ldots, f_{k}\right]$, où la $\mathbb{R}$-sous-algèbre $\mathbb{R}\left[t_{1}, \ldots, t_{v}\right]^{\mathcal{G}}$ de $\mathbb{R}\left[t_{1}, \ldots, t_{v}\right]$ a été introduite dans la définition 3.2 et les $f_{i}$, pour $i=1, \ldots, k$, sont des polynômes homogènes tous de degré supérieur ou égal à 1.

La dernière assertion permet d'aboutir à un système d'équations polynomiales en les coefficients de la matrice des moments qui sont eux-mêmes des polynômes en les coordonnées des points support du plan. Il s'agit donc d'une condition qui peut s'exprimer à l'aide du second objectif aussi bien dans le cas de la construction que de l'augmentation. L'appartenance à un domaine expérimental caractérisé par les inéquations polynomiales $g_{1}(\boldsymbol{x}) \leqslant 0, \ldots, g_{u}(\boldsymbol{x}) \leqslant 0$ est une condition du type premier objectif. D'où le résultat annoncé.

\subsection{Construction ou augmentation optimale d'un plan ex- périmental}

Considérons un critère d'optimalité polynomial en les coefficients des points du plan, il s'agit par exemple des critères de $D$-optimalité, de $I$-optimalité, GH96], ou $\phi_{p}$, avec $p$ entier, [Kie74]. Toutefois lors de la recherche de plans optimaux pour un critère orthogonalement invariant, il est possible de restreindre sa recherche à des plans faiblement invariants, GH96] et en particulier dans le cas des critères de $D$-optimalité et de la $I$-optimalité il est possible de supposer que le plan recherché est de surcroît isovariant. Nous renvoyons au paragraphe 5.3 pour plus de détails.

\subsection{Plans partiellement faiblement-invariants et alphabé- tiquement optimaux}

Cette idée vient du constat suivant : lors de la recherche de plans faiblement invariants, suivant la méthode indiquée en 5.1 il est possible si nous obtenons un ensemble de solutions réelles $\mathbf{V}(I)$ non vide que celui-ci comporte plusieurs solutions voire une infinité. Le premier exemple de ce type que nous avons rencontré, Ber08b], est l'ensemble infini de plans $\boldsymbol{\xi}_{11}(s)$, dont la partie sphérique est de cardinal $r=11$, isovariants pour un modèle polynomial de degré 1 , et non déductibles l'un de l'autre par une transformation orthogonale. Or il n'existe pas de plan isovariant pour un modèle polynomial de degré 2 dont la partie sphérique est de cardinal inférieur ou égal à 12 . Toutefois il est possible d'analyser les plans $\boldsymbol{\xi}_{11}(s)$, complétés par un essai à l'origine, avec un modèle polynomial complet d'ordre 2. Nous ne pouvons pas alors appliquer les résultats de Hardin et Sloane qui nous assureraient que tous les plans $\boldsymbol{\xi}_{11}(s)$, complétés par un essai à l'origine, minimisent les critères de $D$-optimalité et de $I$-optimalité parmi les plans comportant une partie sphérique de cardinal 11 et 1 essai à l'origine. Par conséquent nous sélectionnons parmi cette famille de plans celui qui minimise le critère de $D$-optimalité. Il s'avère alors qu'il minimise tous les critères d'optimalité alphabétique parmi les plans de cette famille. 
La formulation en termes de problème de construction polynomiale, introduit dans la définition 5.1. est la suivante. Résoudre en premier les objectifs 1 et 2 associés aux contraintes sur le domaine expérimental et la formulation polynomiale de l'invariance faible. Étudier le comportement des critères d'optimalité polynomiaux sur l'ensemble des solutions obtenues. Nous pouvons également nous servir de la progammation semi-définie positive, Par03], pour résoudre ce problème.

De la même manière nous pouvons chercher s'il est possible d'augmenter un plan existant pour qu'il vérifie ou continue à vérifier ces propriétés.

\subsection{Plans bloqués orthogonalement}

Il s'agit d'une possible extension de la méthode proposée ici puisque les relations de blocage orthogonal sont également polynomiales en les coordonnées des points support du plan, voir par exemple [BD88] pour plus de détails à ce sujet. Pour certains dispositifs de forme simple, les conditions de blocage orthogonal et d'isovariance ne sont pas nécessairement compatibles pour des modèles polynomiaux complets de degré supérieur ou égal à 2 , voir Mon01. La démarche alors adoptée est souvent la suivante : réaliser le blocage orthogonal avec un faible défaut d'isovariance ou l'isovariance avec un faible défaut de blocage orthogonal. Ce défaut d'isovariance peut être caractérisé par la discrépance du dispositif, voir [HS96], qui est un critère polynomial en les coordonnées des points support du plan, ou tout autre mesure polynomiale en les coordonnées des points support du plan. Il est ainsi possible de chercher des plans bloqués orthogonalement de discrépance minimale à l'aide de la programmation semi-définie positive, $\mathrm{Par03}$.

\section{Méthodologie de résolution}

Des techniques liées aux bases de Gröbner, KR00 et KR05, ou à la programmation semi-définie positive, Par03, permettent de résoudre de tels problèmes de construction qui sont associés au théorème des zéros réels tel que Stengle l'a formulé en 1974, Ste74. Par résolution nous entendons soit l'obtention des coordonnées exactes des points support du plan soit la preuve qu'il n'existe pas de dispositif satisfaisant aux objectifs spécifiés. Cette preuve est une identité polynomiale appelée réfutation et qu'il est possible de déterminer algorithmiquement, Par03. Chercher des plans qui sont une union d'ensembles de points obtenus comme orbite par des groupes de Coxeter, Kan01, diminue le nombre d'inconnues intervenant dans le problème de construction que nous considérerons et augmente de ce fait grandement nos chances d'obtenir une résolution algorithmique de ce problème en un temps acceptable. En effet, les bases de Gröbner, KR00, KR05, qui sont des outils adaptés pour résoudre ces systèmes d'équations polynomiales ont un coût doublement exponentiel.

Ainsi par exemple, pour obtenir un plan isovariant, il suffit de déterminer quelles sont les coordonnées des points dont nous devons prendre l'orbite par un groupe fini. C'est cette stratégie, guidée par des résultats de Hardin et Sloane, [HS96, dont nous nous sommes servis efficacement pour résoudre les problèmes de construction de type polynomial dans [Ber08b]. Néanmoins, lorsque nous nous servons de la théorie de l'élimination et des bases de Gröbner, nous trouvons 
les solutions complexes du problème de construction. Or, nous ne nous sommes intéressés que par les solutions réelles puisque seules celles-ci seront utilisées comme coordonnées des points dont il faudra prendre l'orbite pour construire le support d'un plan solution. Une seconde manière de résoudre ce problème passe par la combinaison de la théorie des bases de Gröbner et de la théorie de la programmation semi-définie positive. Cette approche permet non seulement de déterminer si une solution existe mais aussi de sélectionner parmi toutes les solutions possibles celle qui minimise un critère de type polynomial. Ainsi nous pourrons, par exemple, combiner la résolution des équations d'isovariance à la recherche d'un plan optimal pour un critère d'optimalité qui puisse s'écrire de manière polynomiale.

Nous développons des exemples d'utilisation de cette methodologie dans la prépublication [Ber08b].

\section{Conclusion et perspectives}

Nous avons proposé un cadre algébrique permettant de décider de l'existence d'un plan expérimental satisfaisant à certaines contraintes d'un intérêt majeur pour l'expérimentateur. Celles-ci peuvent porter sur la localisation des points support du plan, sur une propriété d'invariance faible qui doit être vérifiée par le plan ou sur une condition de blocage orthogonal. Parmi tous les plans solutions il est également possible de déterminer celui qui minimise un critère polynomial en les coordonnées des points support du plan et donc de sélectionner des plans qui sont de surcroît alphabétiquement optimaux. L'introduction de ce cadre algébrique permet d'avoir recours à une résolution algorithmique du problème de construction ce qui permet généralement de déterminer les coordonnées des points support du plan de manière exacte. Il est alors possible d'utiliser les techniques relevant de la statistique algébrique, [PRW00], pour analyser ces dispositifs. La détermination exacte des confusions d'effets, l'obtention d'un modèle saturé ou les tests de défaut d'ajustement font partie des outils supplémentaires alors à la disposition de l'expérimentateur.

Cette méthodologie a été appliquée à la construction de plans sphériques isovariants dans $\mathbb{R}^{3}$, Ber08b], et a servi à démontrer plusieurs résultats d'existence de plans sphériques isovariants dont les coordonnées des points support du plan sont connues de manière exacte. Le cas de la dimension 4 est en cours d'étude, s'appuyant cette fois-ci sur l'étude numérique préalable débutée dans [SHC03]. Le calcul de réfutations permettant de démontrer une conjecture de Hardin et Sloane, [HS96], fera l'objet d'un prochain article.

\section{Références}

[BD88] G.E.P. Box and N.R. Draper. Empirical model building and response surfaces. Wiley, New York, 1988.

[Ber08a] F. Bertrand. Isovariance et $\mathcal{G}$-invariance faible en planification expérimentale. Prépublication de l'IRMA, 2008. http ://hal.archives-ouvertes.fr/hal-00276011/fr/.

[Ber08b] F. Bertrand. Problèmes de construction de type polynomial II - Quelques résultats d'existence de plans sphé- 
riques isovariants exacts. Prépublication de l'IRMA, 2008. http ://hal.archives-ouvertes.fr/hal-00278267/fr/.

[BH57] G.E.P. Box and J.S. Hunter. Multi-factor experimental designs for exploring response surfaces. Annals of Mathematical Statististics, $28: 195-241,1957$.

[BOB07] F. Bertrand, A. Ourliac, and B. Boulanger. Recherche numérique de plans $d$-optimaux pour des problèmes de pharmacocinétique et pharmacodynamique : une étude de cas. Actes des Journées de la Statistique organisée par la Société Française de Statistique, 2007.

[CMMR87] A. Corana, B.L. Marchesi, C. Martini, and S. Ridella. Minimizing multimodal functions of continuous variables with the simulated annealing algorithm. ACM Transactions on Mathematical Software, $13: 262-280,1987$.

[DMP05] H. Dette, V.B. Melas, and A. Pepelyshev. Optimal designs for three-dimensional shape analysis with spherical harmonic descriptors. Annals of Statistics, 33 :2758-2788, 2005.

[Far06] J. Faraut. Analyse sur les groupes de Lie. Calvage \& Mounet, Paris, 2006.

[GH96] N. Gaffke and B. Heiligers. Approximate designs for polynomial regression : Invariance, admissibility and optimality. In S. Ghosh and C.R. Rao, editors, Handbook of Statistics, volume 13, chapter 30, pages 1149-1199. Elsevier Science B.V., 1996.

[GW00] J. Gabrielsson and D. Weiner. Pharmacokinetic and Pharmacodynamic Data Analysis : Concepts and Applications,. Swedish Pharmaceutical Press, 2000.

[Hil82] H. Hiller. Geometry of Coxeter groups, volume 54 of Research Notes in Mathematics. Pitman, Boston, 1982.

[HS91] R.H. Hardin and N.J.A. Sloane. Operatig Manual for Gosset : A general-Purpose Program for Constructing Experimental Designs, volume 98 of Statistics Research Report. AT\&T Bell Labs, Murray Hill, second edition, 1991.

[HS96] R.H. Hardin and N.J.A. Sloane. Mclaren's improved snub cube and other new spherical designs in three dimensions. Discrete Compu. Geom., 15 :429-441, 1996.

[Hum90] J.E. Humphreys. Reflection groups and Coxeter groups, volume 29 of Cambridge Studies in Advanced Mathematics. Cambridge University Press, 1990.

[Kan01] R.M. Kane. Reflection groups and invariant theory. Number 5 in CMS books in mathematics. Springer-Verlag, New-York, 2001.

[Kem93] S. Kemper. The invar package for calculating rings of invariants. IWR Preprint Universitat Heidelberg, 93-94., 1993.

[Kie74] J. Kiefer. General equivalence theory for optimum designs (approximate theory). Annals of Statistics, 2 :849-879, 1974.

[KR00] M. Kreuzer and L. Robiano. Computational Commutative Algebra 1. Springer, Berlin-Heidelberg, 2000. 
[KR05] M. Kreuzer and L. Robiano. Computational Commutative Algebra 2. Springer, Berlin-Heidelberg, 2005.

[Mon01] D.C. Montgomery. Design and Analysis of Experiments. Wiley, New York, fifth edition, 2001.

[MT86] R. Mneimné and F. Testard. Introduction à la théorie des groupes de lie classiques. Hermann, Paris, 1986.

[NM92] N.-K. Nguyen and A.J. Miller. A review of some exchange algorithms for constructing discrete d-optimal design. Computational Statistics $\& 3$ Data Analysis, 14 :489-498, 1992.

[Par03] P.A. Parrilo. Semidefinite programming relaxations for semialgebraic problems. Mathematical Programming Ser. B, 96 :293-320, 2003 .

[PRW00] G. Pistone, E. Riccomagno, and H.P. Wynn. Gröbner basis methods for structuring and analysing complex industrial experiments. International Journal of Reliability, Quality and Safety Engineering, 7 :285-300, 2000 .

[Puk93] F. Pukelsheim. Optimal Design of Experiments. Wiley, New-York, 1993.

[SHC03] N.J.A. Sloane, R.H. Hardin, and P. Cara. Sperical designs in four dimensions. Extended Abstract, 2003.

[Ste74] G. Stengle. A nullstellensatz and a positivstellensatz in semialgebraic geometry. Math. Ann., 207 :87-97, 1974.

[Stu02] B. Sturmfels. Solving Systems of Polynomial Equations, volume 97 of Conference Board of the Mathematical Sciences : Regional Conference Series in Mathematics. American Mathematical Society, 2002.

[Ven01] B Venkov. Réseaux et designs sphériques. In J. Martinet, editor, Réseaux euclidiens, designs sphériques et formes modulaires : Autour des travaux de Boris Venkov, volume 37 of Monographies de L'Enseignement Mathématique, chapter 1. L'enseignement mathématique, Genève, 2001.

[Wei65] A. Weil. L'intégration dans les groupes topologiques et ses applications. Hermann, Paris, 1965. 